\title{
Angularly Resolved Deep Brain Fluorescence Imaging Using a Single Optical Fiber
}

\author{
Israel J. De La Rosa $\left(\mathbb{D},{ }^{1}\right.$ Mehdi Azimipour, ${ }^{2}$ Patrick K. Cullen, \\ Fred J. Helmstetter, ${ }^{3}$ and Ramin Pashaie ${ }^{1}{ }^{1}$ \\ ${ }^{1}$ Electrical and Computer Engineering Department, University of Wisconsin-Milwaukee, Milwaukee, Wisconsin, 53201, USA \\ ${ }^{2}$ Vision Science and Advanced Retinal Imaging Laboratory (VSRI), UC Davis Eye Centre, Sacramento, California, 95817, USA \\ ${ }^{3}$ Department of Psychology, University of Wisconsin-Milwaukee, Milwaukee, Wisconsin 53201, USA \\ Correspondence should be addressed to Ramin Pashaie; pashaie@uwm.edu
}

Received 9 April 2018; Accepted 17 July 2018; Published 23 August 2018

Academic Editor: Stefan Wabnitz

Copyright (C) 2018 Israel J. De La Rosa et al. This is an open access article distributed under the Creative Commons Attribution License, which permits unrestricted use, distribution, and reproduction in any medium, provided the original work is properly cited.

In this article, we report the development of a minimally invasive fiber optic based fluorescence probe which can reach deep brain objects and measure the intensity and spatial distribution of fluorescence signals in the tissue. In this design, the brain is scanned by a single penetrating side-firing optical fiber which delivers excitation light pulses to the tissue at different depths and orientations and simultaneously collects samples of fluorescence emission signals. Signal-to-noise ratio of the measurements is improved by adapting the pulse compression technique and the theory of optimal filters. Effects of each design parameter on the overall performance of the scanner, including the spatial resolution and speed of scanning, are analyzed and experimentally measured. In vivo experiments show that the new device, despite the simplicity of the design, provides valuable information particularly useful in optogenetic stimulation experiments where the exact position of the fiber tip and the radiation orientation can change the outcome of a test.

\section{Introduction}

Recent advances in the discovery or synthesis of new fluorescent indicators have been followed by the development of sophisticated technologies for in vitro and in vivo fluorescence imaging offering spatial resolution close to the diffraction limit of adapted optical wavelengths [1]. Nonetheless, in vivo fluorescence imaging has remained limited only to the very immediate superficial areas of the brain tissue. A good example of such imaging modalities is the two-photon excitation microscopy, which allows imaging of living tissue up to one millimeter in depth and the system can image a large portion of the cortex in small rodents [1]. Since most high-level information processing operations take place in the cortex, invention of two-photon microscopes opened new horizons in the study of the cortical dynamics. Fluorescence tomography scanners are also made that employ mathematical (e.g., fluorescence diffuse optical tomography (FDOT) [2]) or statistical algorithms (e.g., fluorescence laminar optical tomography (FLOT) $[3,4]$ ) that model light tissue interaction and use this information to translate the measured data to three-dimensional images that reveal the distribution of fluorescent molecules. Usually such tomography scanners can image slightly deeper areas; however, resolution is significantly sacrificed and spurious objects appear in generated images caused by the instability of algorithms that solve ill-posed inverse problems. As a result, none of these technologies can reliably image deep brain objects, such as the thalamus or hippocampus, which play crucial roles in vital brain functions including the early processing of sensory inputs or memory consolidation. For such applications, the main option is to conduct the experiments in brain slices which is an invasive process and certainly nonreversible. Therefore, there is high demand for the invention of new instrumentation that can reach deep brain objects to perform fluorescence imaging.

A reasonable approach to achieve optical access to deep brain regions is the use of thin optical fibers. An optical fiber can function as a reliable tool to guide the power of a coherent or incoherent light source to any target area 

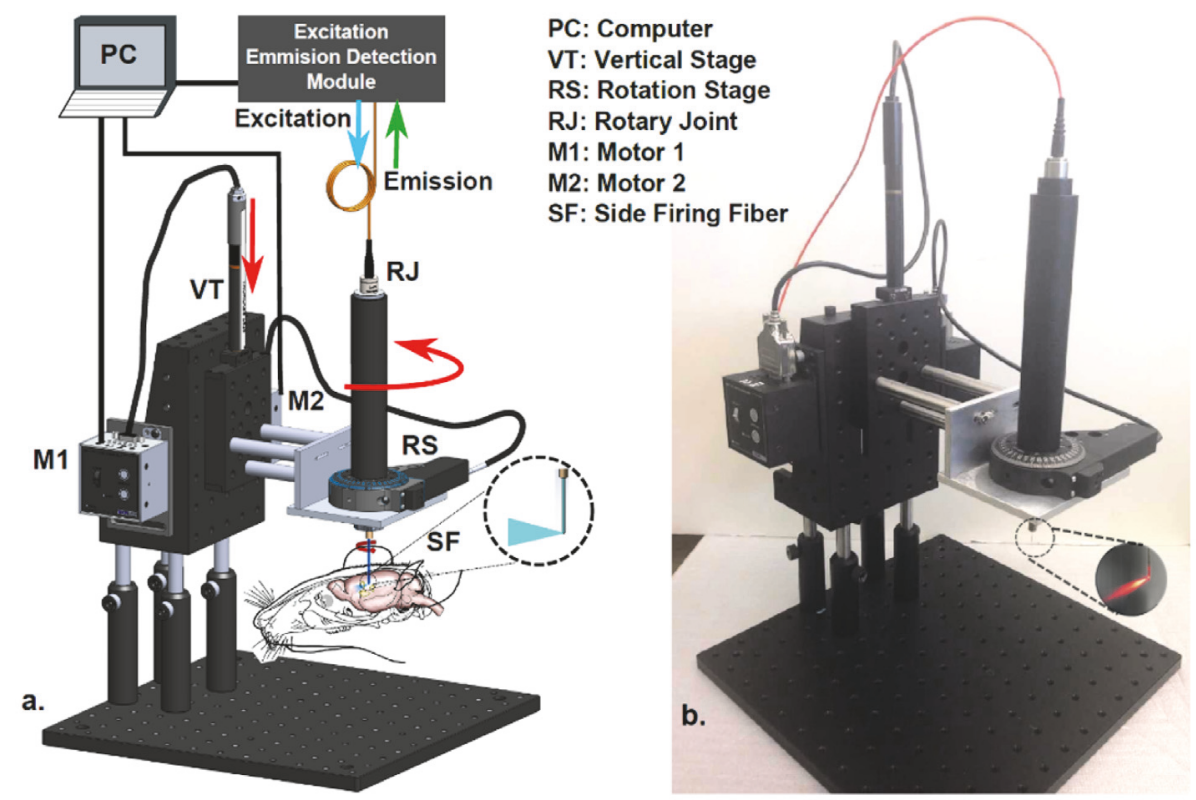

FIGURE 1: (a) Schematic of the setup: two computer controlled actuators adjust the position and orientation of a side-firing optical fiber inside the tissue. A fiber rotary joint is used to help rotate the fiber with no mechanical stress. At each position, the system sends an excitation laser pulse and detects a sample of the corresponding emission signal. Next, the collected data is processed by a computer to generate images that show the distribution of fluorescence signal in a cylinder surrounding the fiber; (b) picture of the developed system.

with minimum loss. Such waveguides are used in many challenging biomedical applications, for example, to perform fluorescent detection (e.g., see $[5,6]$ ) spectroscopy (e.g., see [7-9]) or the assessment of tissue optical property (e.g., see $[8,10,11])$ for optical biopsy applications. The performance of such mechanisms is also theoretically and empirically analyzed in recent years (e.g., see $[12,13])$.

Here, we introduce a new approach in which the brain tissue is scanned by advancing and simultaneously rotating a single side-firing optical fiber inside the tissue. This fiber delivers the excitation light pulses at each site and guides a sample of the emission signal back to the photodetectors. In the brain research applications, we prefer to reduce the size of the optical fiber to minimize the damage to the tissue under test. Reducing the fiber diameter translates to some major reduction in the number of collected photons and as a result, the signal-to-noise ratio dramatically drops in such measurements. We recently introduced a single optical fiber probe for fluorescence measurement and optogenetic stimulation in which the excitation laser pulses were frequency modulated in the form of linear or nonlinear chirped pulses [5, 14]. Next, the system detects the corresponding chirped emission signals and passes them through a correlation filter to compress the energy of the pulse and momentarily maximize signal-tonoise ratio. Since the noise of the system was estimated to be white Gaussian, the correlation filter functions as the optimal filter and helps to detect the most fainted fluorescence signals. The balanced photodetection mechanism adapted in the platform also helps to remove the effect of systematic distortions including the instability of the excitation laser or noise of the detectors [5]. Here, this fluorescence detection module (FDM) is connected to the new head in which the tissue is scanned by the rotating side-firing optical fiber. Details of the design and results from computer simulations and experimental work are discussed in the article.

\section{Materials and Methods: Design and Components}

The main component of the new scanner is the side-firing fiber. A side-firing fiber is an optical fiber in which the delivery end is angled to deflect the fiber's radiation profile. When designed correctly, the main radiation lobe of the sidefiring fiber is almost orthogonal to the fiber's optical axis which is an ideal factor in the design of the probe. Nonetheless, electromagnetic analysis of the structure shows that the divergence of the beam increases as the radiation angle gets closer to $90^{\circ}$ with respect to the fiber's axis. Therefore, there is a trade-off between the direction and directivity of radiation. The appearance of sidelobes in the radiation profile of sidefiring fibers, which causes considerable light power leaking out in unintended directions, is another design concern.

We have proved mathematically and experimentally that by selecting the right value for the tip angle, based on the refractive index and numerical aperture of the fiber, we can design an optimal side-firing fiber for which (1) total internal reflection occurs for all incident rays hitting the angled tip and as a result no leakage occurs, (2) the radiation angle is close to normal, and (3) beam divergence is minimized to help improve the spatial resolution of the scanner [5].

The proposed system is shown in Figure 1 and includes a custom made high hydroxyl ion (high $\mathrm{OH}$ ) silica core sidefiring fiber (Polymicro Technologies, IL, USA), rotation arm (rotary joint, lens tube, and fiber chuck), two servo motor 
actuators with their corresponding servo motor controllers, structural components needed to hold the system together, a laptop computer, and the FDM system. The FDM system is not shown here but the schematic of the design is available in Figure 1 of [5]. Motor controllers, M1 and M2, drive the vertical and rotational translation stages, VT and RS, to precisely position the optical fiber at all target coordinations while the FDM delivers excitation light pulses and detects fluorescence emission through the side-firing fiber, SF. For the side-firing optical fiber that we used in this research, we measured the numerical aperture experimentally and we found NA $=0.34$ in air at room temperature. Our measurements also showed that the fiber radiates the optical power at $73.81^{\circ}$ angle with respect to the optical axis of the fiber. As a result, the main radiation lobe is not completely orthogonal to the fiber and this effect needs to be compensated during image reconstruction process.

\section{Materials and Methods: Design Considerations}

The rotary joint, $R J$, allows the fiber to rotate without twisting that can affect or even damage the waveguiding property of the fiber. The stationary side of the rotary joint is connected to the optical fiber that guides light pulses from the FDM to the scanner's head. The rotating side of the rotary joint holds the side-firing fiber that is mounted in a fiber chuck (HFR001, Thorlabs, NJ, USA) and positioned within the mechanism of the rotary servo motor. We use FC/PC to FC/PC mating sleeves to connect the fibers to the joints. It is important to make the fiber rotate completely along its axis. Aligning the optical fiber is a vital step and it needs to be adjusted sequentially three set screws, one revolution at a time, until the chuck that holds the fiber is firmly secured. If this is not done properly, the tip of the fiber will follow an elliptical trajectory that is quite destructive inside the soft tissue of the brain; see Figure 2(a).

Another important consideration for the rotary joint is the fluctuations in the coupling of the light from the stationary fiber to the side-firing fiber as a function of the angular position of the tip. To estimate this effect, we connected the stationary fiber to a stable source (M47051, 470nm fiber coupled LED, Thorlabs, NJ, USA) while we were measuring the light power radiating from the rotating fiber by an optical power sensor (S130C, Thorlabs, NJ, USA). We repeated the experiment six times and averaged the data. Results are shown in Figure 2(b). Left panel displays the normalized coupling factor as a function of the position and orientation of the tip. From these results, we noticed that the rotary joint had a tendency to increase the detection intensity at the angle of approximately $150^{\circ}$. The maximum amplitude of these fluctuations compared to the base line of the measured power is small; nonetheless, such tiny distortions can still affect the outcome particularly when we are measuring faint fluorescence signals. To compensate this effect, we generated a complementary map, shown in Figure 2(b) right panel, and used that to normalize the collected data and eliminate this effect.

\section{Results and Discussion}

4.1. Simulation Results. To better understand and analyze the performance of this scanner, we used the stochastic method of Monte Carlo (MC) $[15,16]$ to simulate light propagation in a scattering and absorbing medium. Based on our previous experience of running these simulations for light brain tissue interaction [17], we chose to launch and trace $5 \times 10^{6}$ photons in the tissue which seems to be enough to generate a good estimation of how many photons find their way back to the fiber tip for different parameters of the medium, optical fiber, or wavelengths. Scattering coefficient of the brain tissue was modelled by $\mu_{s}^{\prime}=a(\lambda / 500 \mathrm{~nm})^{-b}$, where $\lambda$ is the excitation or emission wavelength and $a$ is a scaling factor which represents the reduced scattering coefficient at $\lambda=$ $500 \mathrm{~nm}$. The parameter $b$ in this equation is the scattering power. Values for $a$ and $b$ are $2.37 \mathrm{~mm}^{-1}$ and 1.15 , respectively [15]. This calculation gives $\mu_{s}^{\prime}=23.2 \mathrm{~cm}^{-1}$. The absorption coefficient, $\mu_{a}$, was calculated by $\mu_{a}=B S \mu_{\mathrm{HbO}_{2}}+B(1-S) \mu_{\mathrm{Hb}}+$ $W \mu_{\text {water }}$. Here, $B$ is the average blood volume fraction, $S$ is the hemoglobin oxygen saturation, and $W$ is the water content. The values for $B, S$, and $W$, are $0.028,0.62,0.62$, respectively [15]. The absorption coefficients for hemoglobin and water were taken from the Oregon Medical Laser Center website [18]. This calculation gives $\mu_{a}=3.36 \mathrm{~cm}^{-1}$. The value of $\mathrm{g}$ was set to 0.9 and the value of $\mu_{s}=\mu^{\prime}{ }_{s} /(1-\mathrm{g})=232.0 \mathrm{~cm}^{-1}$.

We used these values to define a scattering and absorbing medium in the MC simulation software (https://omlc .org/software $/ \mathrm{mc} /$ ) and we modelled the performance of the scanner when a $250 \mu \mathrm{m}$ diameter fluorescent particle, emitting $510 \mathrm{~nm}$ wavelength, was placed at the radial distance of $0.5 \mathrm{~mm}$ from the fiber tip. First, we looked at the effect of the fiber's $N A$ on the spatial resolution of the scanner. Figure 3 shows the simulation results when the $N A$ varies from 0.2 to 0.6 . In each test, we computed the full-width halfmaximum (FWHM) of the image of the particle produced by the scanner as a measure of the resolution. The left panel in this figure shows the FWHM along the vertical axis and the right panel displays the radial FWHM. From these plots, we observe that increasing the NA translates to some noticeable increase in the FWHM or reduction of the spatial resolution. Therefore, we prefer side-firing fibers that offer smaller numerical apertures; however, reducing the divergence of the radiation cone for a side-firing fiber usually comes with the main lobe of radiation being less orthogonal to the optical axis of the fiber. As a result, there is a tradeoff in this design. The dashed line in these graphs represent $N A=0.34$ which is the numerical aperture of the side-firing fiber we used for our experiments and as mentioned before the beam makes the angle of $73.81^{\circ}$ with respect to the fiber's optical axis.

4.2. Phantom Results. To test the precision of our simulations, we built eight tissue phantoms. The phantoms were made by suspending a $250 \mu \mathrm{m}$ fluorescence microsphere (using an ultra-thin wire and optically clear epoxy (Loctite)) in liquid mixtures of milk and India ink in different concentrations to achieve any combination of scattering and absorption 

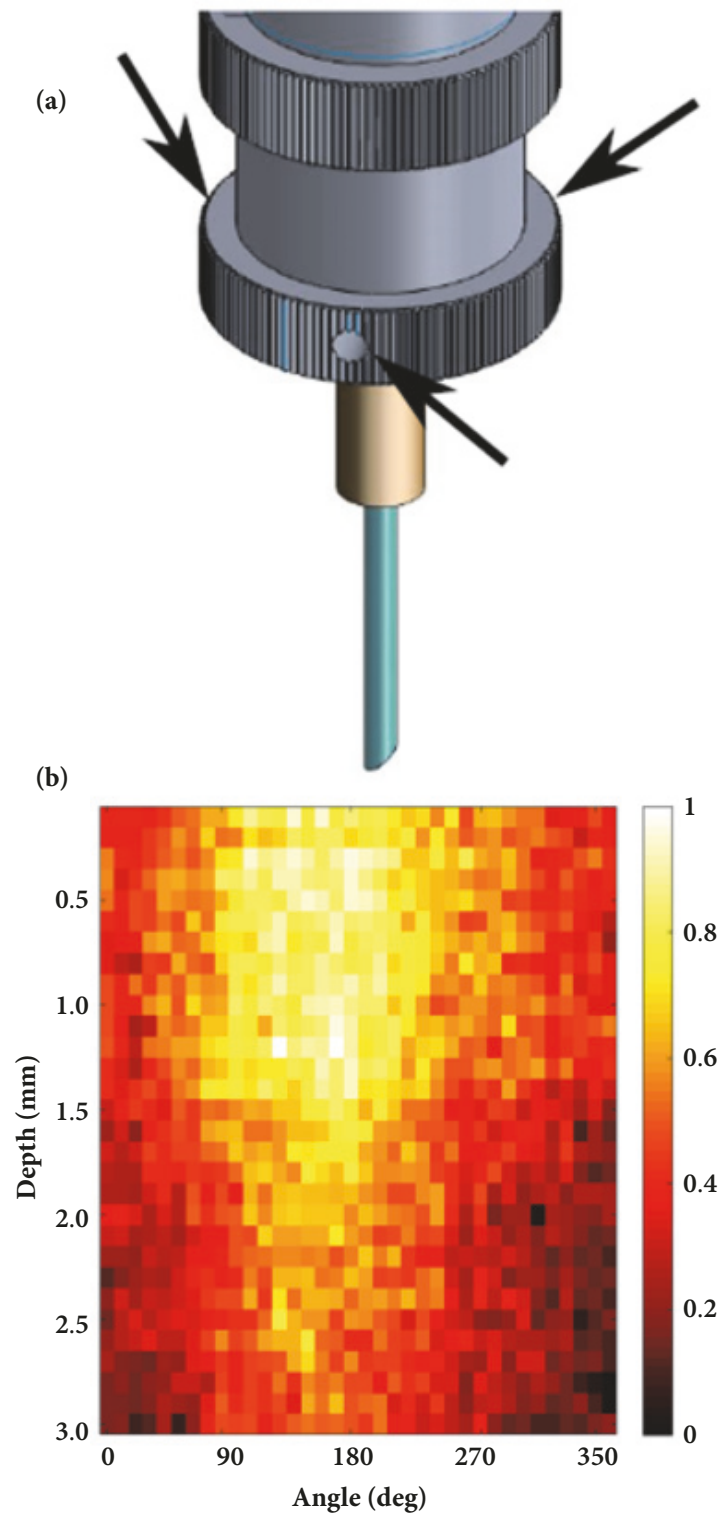

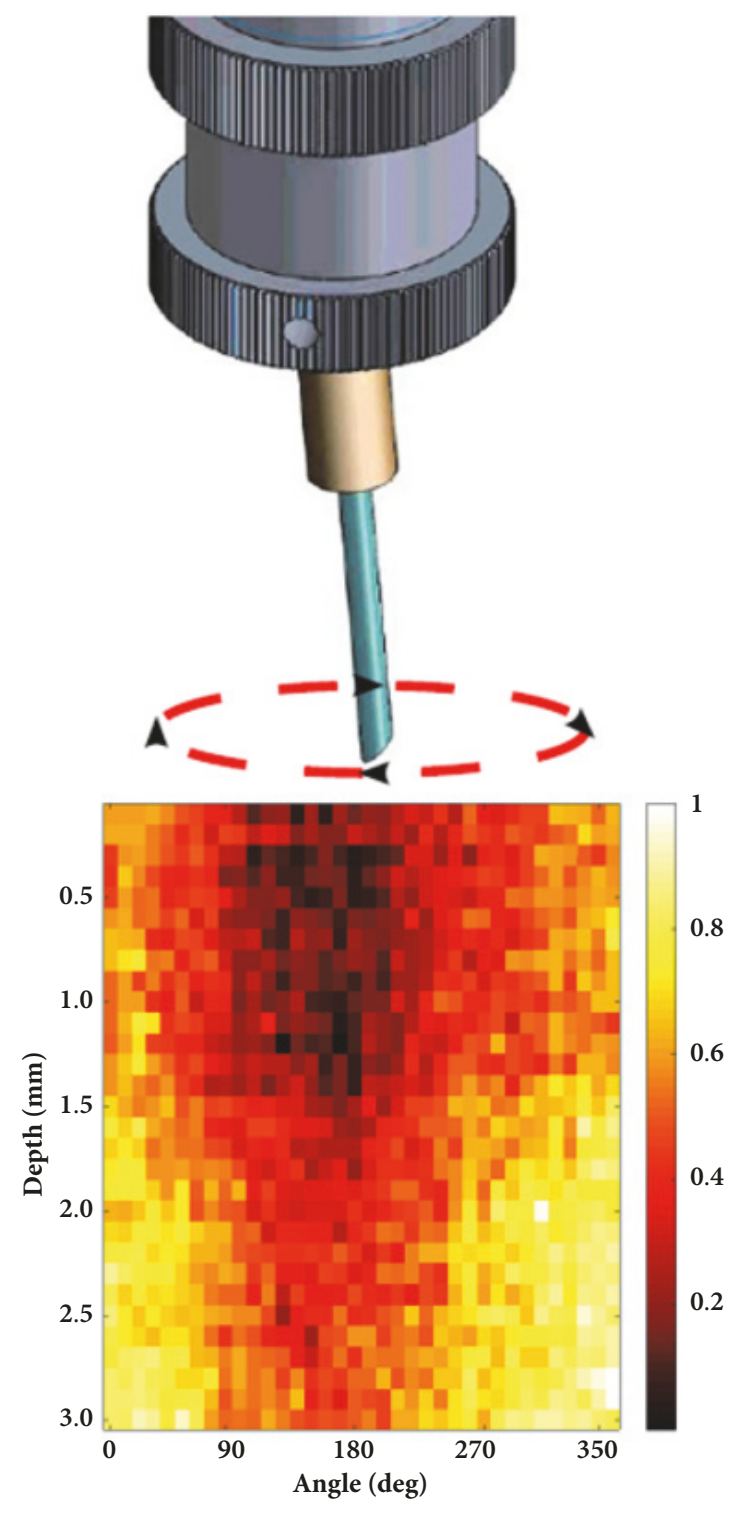

Figure 2: (a) Arrows point to the position of the set screws that need to be adjusted to keep the chuck aligned along the vertical axis (Left). Undesired trajectory of optical fiber resulting from improper set screw adjustment (Right). (b) Fluctuations caused by the rotary joint (left) introduce some nonuniformity in fluorescence intensity recording which in this case shows a spike at 150 degrees. To eliminate the effect, this nonuniformity was measured in a uniform fluorescence solution. Next, the complement of this data was created as a distortion compensation matrix (Right) and this matrix was used to cancel the effect.

coefficients. We scanned these phantoms one by one and we compared the measured resolution with the estimated values from simulations; see Figure 4. Based on this comparison, the measured FWHMs are within $20 \%$ of the simulation results when the scattering coefficient was fixed but the absorption was changing over a reasonable range. On the other hand, when we were changing the scattering coefficients for a medium with fixed absorption, the results were within $25 \%$ of the simulated results which are still reasonable. The resolution of optical imaging systems is limited because of scattering in the tissue. The impact of absorption coefficient is not more than reducing the absolute signal. Figure 4 shows the higher impact of reduced scattering on the resolution compared to the effect of absorption on the resolution.

Simulations provide an intuition to the response of the system when making measurements in the tissue. One important parameter for in vivo experiments is the tradeoff between the speed of scanning and the resolution of the reconstructed images. Reducing the vertical or radial distance between the measurement points potentially leads to better resolution but makes the scanning process highly time consuming. For instance, scanning with axial and radial resolutions of $100 \mu \mathrm{m}$ and $10^{\circ}$ and taking two measurements for each point can take up to $45 \mathrm{~min}$, which is too long. We 

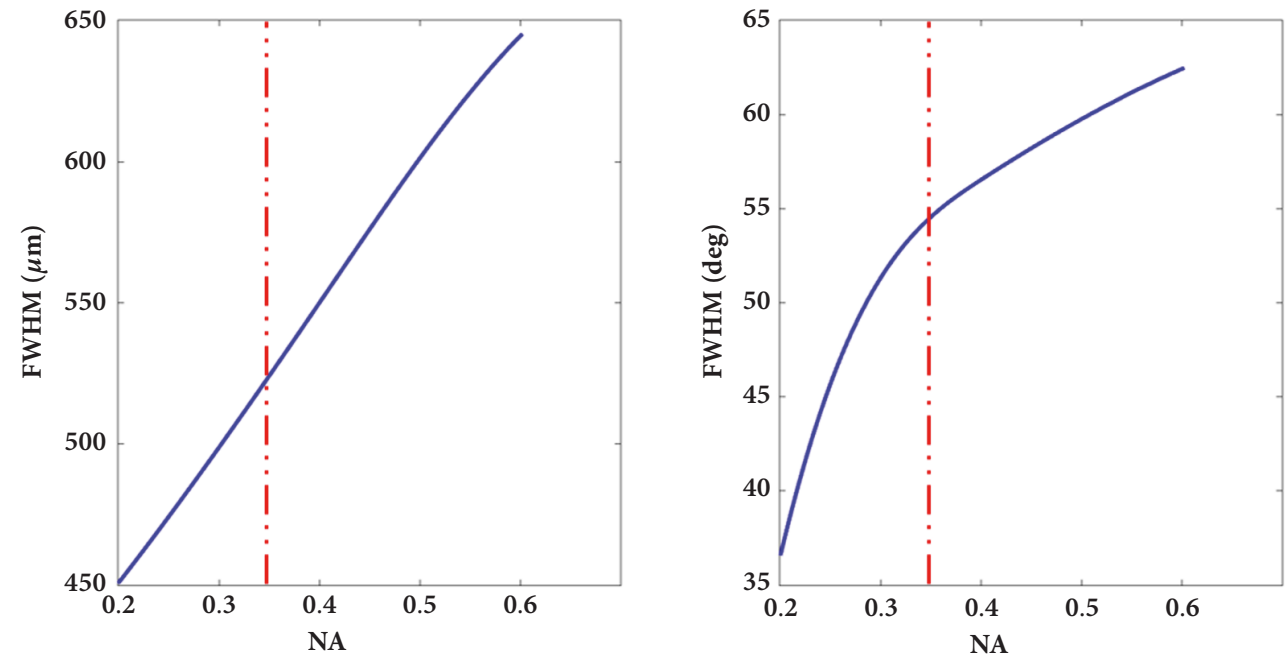

FIGURE 3: Plots of the effects from a varying NA on the FWHM for the fluorescent of a $250 \mu \mathrm{m}$ particle. Left plot shows the behavior of the FWHM vertically while right plot shows the behavior radially. The red lines represent the $N A=0.34$ which is the NA of the optical fiber in our system.
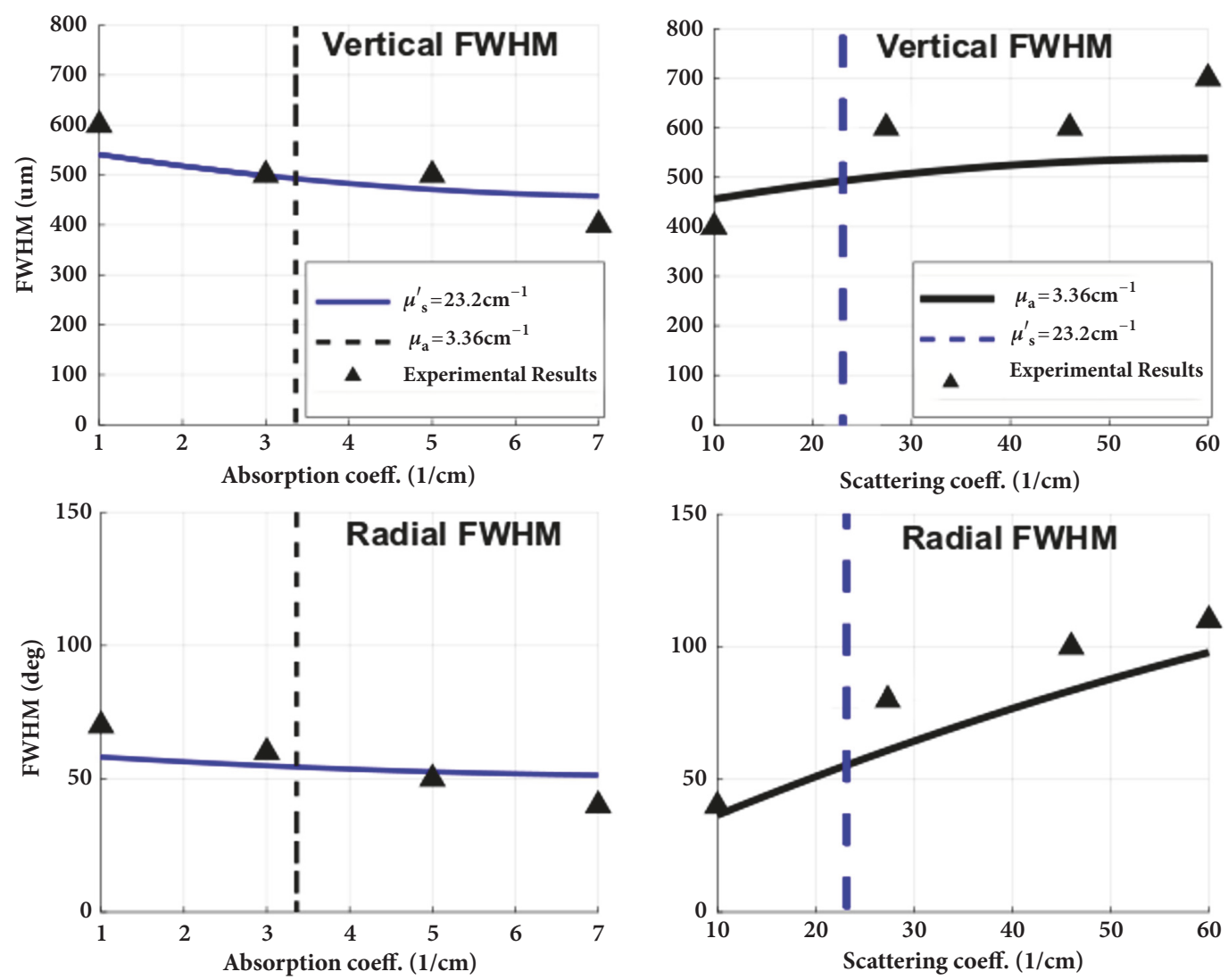

FIGURE 4: Results from phantom experiments compared to simulations measuring resolution in vertical and radial direction. For the absorption coefficient, $\mu_{a}$, four data points were collected at 1.0, 3.0, 5.0, 7.0 $\mathrm{cm}^{-1}$. Our estimation for the value of $\mu_{a}$ in brain tissue is 3.36 $\mathrm{cm}^{-1}$. Phantom results are within $20 \%$ of our simulations (Left). For the reduced scattering, $\mu_{s}^{\prime}$, four data points at $10.0,26.0,43.0,60.0 \mathrm{~cm}^{-1}$ were collected. The value of $\mu_{s}^{\prime}$ we used for simulations was $23.2 \mathrm{~cm}^{-1}$. These results are within $25 \%$ of the simulated values for the FWHM. 


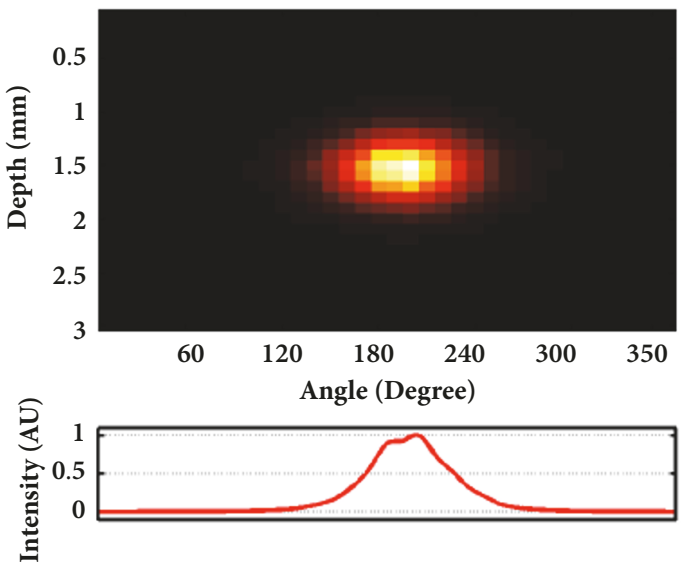

(a)
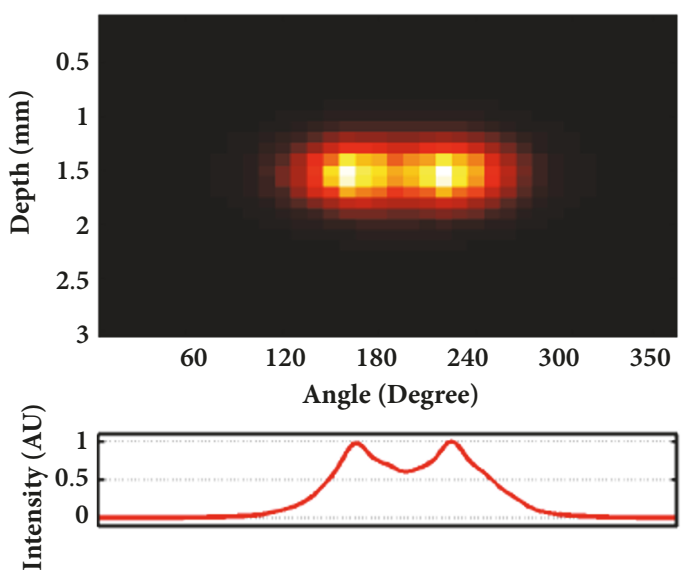

(c)

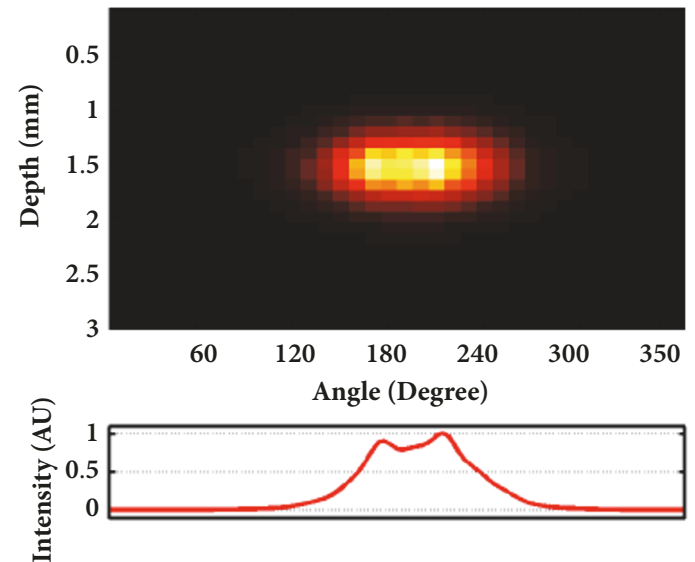

(b)
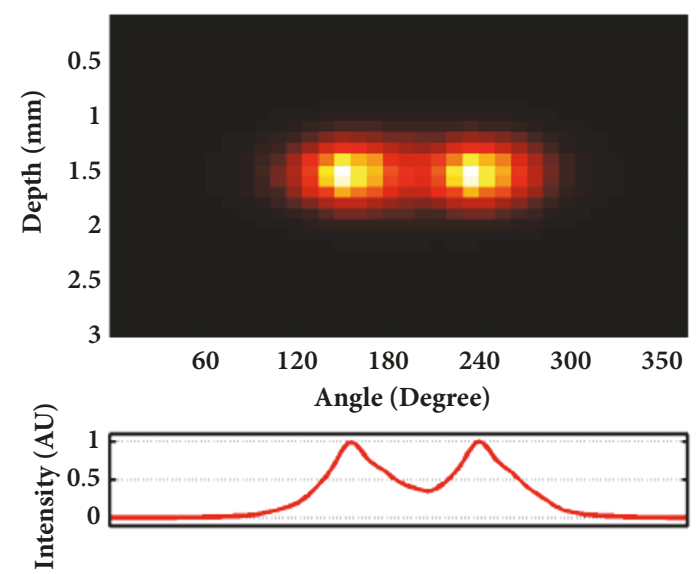

(d)

Figure 5: Results from a simulation to find the resolution of our proposed system. Based on this result, when the particles are positioned at the distance of $0.5 \mathrm{~mm}$ from the fiber tip, the particles are still detectable as two separate particles and the angular distance between them is larger than $50^{\circ}$.

also know that, for a fiber with a given NA, reducing the distance between the points of measurements will not even provide more information and the collected data will become redundant. Figures 5 and 6 show the results of our simulation where we placed two $250 \mu \mathrm{m}$ diameter fluorescence particles $0.5 \mathrm{~mm}$ apart from the tip of the side-firing fiber and we imaged them while changing the vertical or horizontal distance between the particles. In these simulations, photons were launched uniformly within the diameter of the optical fiber used in our system $(200 \mu \mathrm{m})$ and the corresponding NA (0.34). The optical properties used were $\mu$ 's $=23.2 \mathrm{~cm}-1$, $\mu \mathrm{a}=3.36 \mathrm{~cm}-1$, and $\mathrm{g}=0.9$. In these figures we can see the reconstructed images when the particles are placed at the horizontal and vertical distances of $0.3 \mathrm{~mm}$ to $0.6 \mathrm{~mm}$ from each other.

It is clear from the figures that the particles are not distinguishable when the distance between them is less than $0.5 \mathrm{~mm}$. This means that the rotation angle needs to be less than $50^{\circ}$. Nonetheless, $50^{\circ}$ is still too large since, for the $50^{\circ}$ angular scanning step size, one of these particles can be missed. Therefore, the limit for detecting particle of this size at this distance is half of $50^{\circ}$. In Figure 6 we can see that the two particles are not distinguishable as separate particles when the vertical distance between them is less than $0.49 \mathrm{~mm}$. Consequently, the vertical steps need to be at least half of this value if the goal is to detect particles of this size $(250 \mu \mathrm{m})$ at this distance $(0.5 \mathrm{~mm})$. In Figure 7 axial and angular resolutions for the same particle size are plotted as a function distance from the fiber tip. In most in vivo experiments, we use the scanning angle of $20^{\circ}$ and the vertical scanning steps of $0.2 \mathrm{~mm}$.

Another consideration is changing the wavelength of the system. By simulating multiple wavelengths, Figure 8, we see that changing the wavelength affects the resolution of the system. As the wavelength increases, the scattering coefficient for the brain tissue decreases but so does the absorption coefficient. Since the tissue does not absorb many photons for longer wavelengths, the smaller number of photons that do scatter affects the FWHM of an imaged particle and the resolution of the system. 


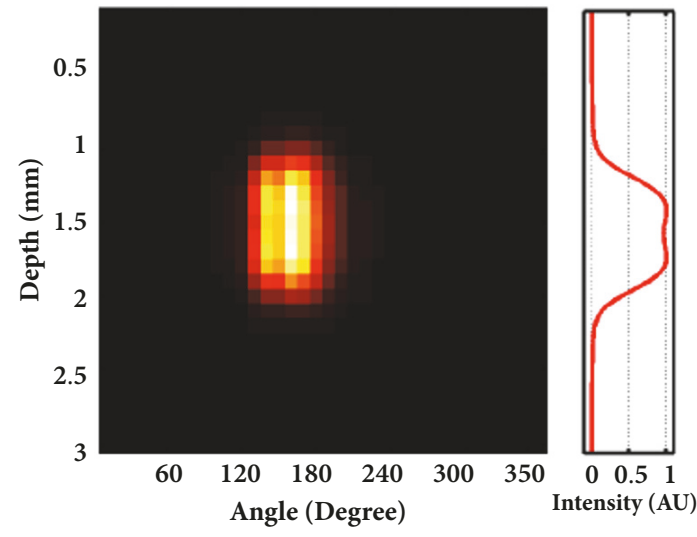

(a)

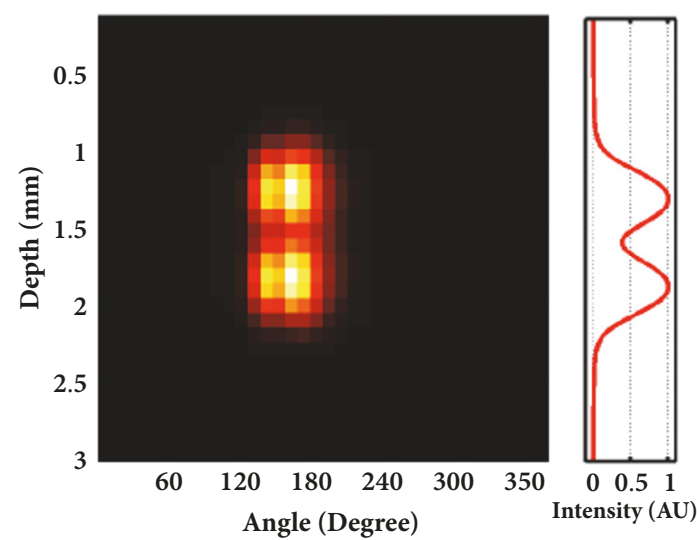

(c)

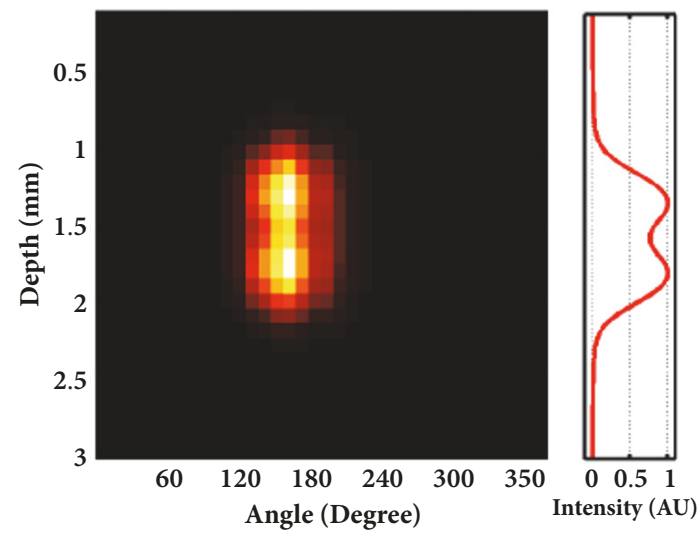

(b)

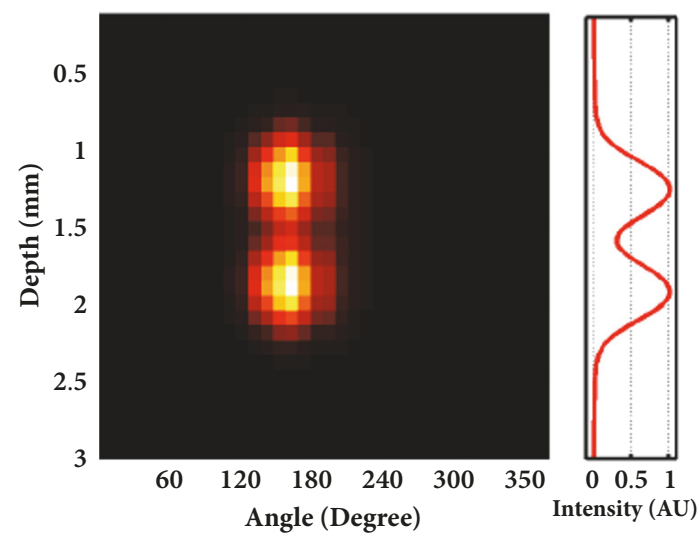

(d)

FIGURE 6: Results from a simulation to find the resolution of our proposed system. Based on this result, when the particles are positioned at the distance of $0.5 \mathrm{~mm}$ from the fiber tip, the particles are still detectable as two separate particles and the vertical distance between them is larger than $0.49 \mathrm{~mm}$.

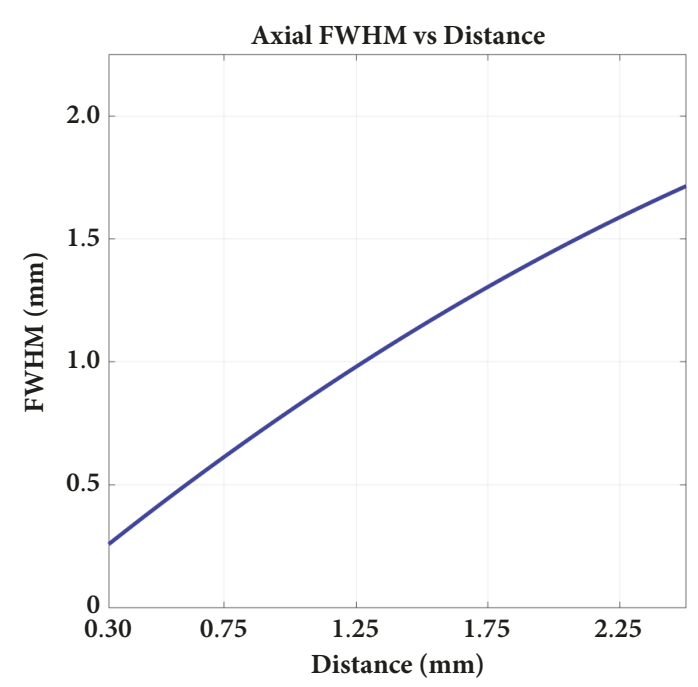

(a)

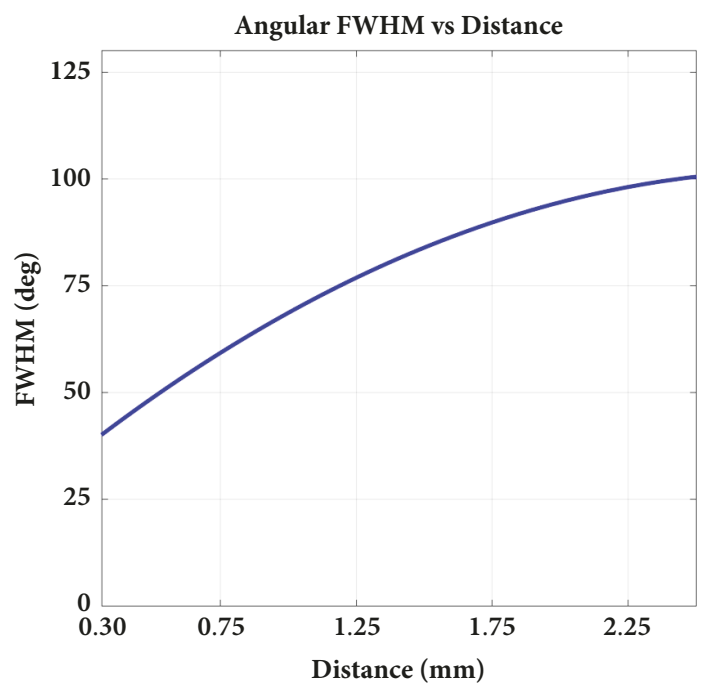

(b)

FIGURE 7: Axial (a) and angular (b) resolutions displayed as a function of distance from the fiber tip. Resolution is measured as the FWHM of a $250 \mu \mathrm{m}$ fluorescence particle. 


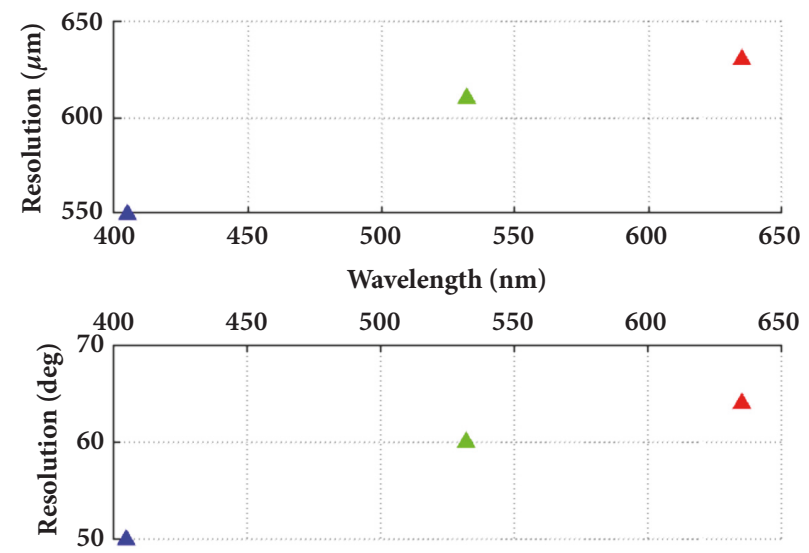

FIGURE 8: Results from spectral response simulations. Increasing the wavelength has a negative effect on the resolution of the proposed system. Resolution is measurement in terms of the FWHM of the imaged fluorescence particle.

4.3. In Vivo Results. To test the performance of the system when imaging the brain of a live animal, we used a male Long-Evans rat (300g-400g, Envigo, WI). All procedures were carried out in a facility accredited by the Association for Assessment and Accreditation and Laboratory Animal Care (AAALAC) and approved by the University of WisconsinMilwaukee Institutional Animal Care and Use Committee (IACUC) and conducted within the ethical guidelines of the National Institutes of Health (NIH). Two weeks prior to the experiments, the animal was anesthetized with isoflurane in $100 \%$ oxygen (induction occurred with $4 \%$ isoflurane and maintained with $2 \%$ ). The rat was mounted in a stereotaxic apparatus (Kopf Instruments, CA, USA). Purified AAV9CAG-GFP virus (UNC Vector Core) was infused into ventral hippocampus (A/P $-5.28 \mathrm{~mm}, \mathrm{M} / \mathrm{L}+5.4 \mathrm{~mm}, \mathrm{D} / \mathrm{V} 5.7 \mathrm{~mm})$ to express green fluorescence protein (GFP) in the tissue. The injection volume was $0.3 \mathrm{ml}$. The virus was infused using a $10 \mu \mathrm{l}$ nanofil syringe and 34-gauge needle (World Precision Instruments) mounted to a stereotaxic automated injector (Micro4 MicroSyringe Injector; World Precision Instruments) at a rate of $0.05 \mathrm{ml} / \mathrm{min}$. The injector was left in place for 10min following the injection to allow diffusion of the virus away from the injector.

The experiment was conducted by scanning the brain tissue starting at the depth of $4.4 \mathrm{~mm}$ inside the tissue and exactly at the coordination of the viral injection. Then, we scanned the brain tissue with the side-firing optical fiber using the $20^{\circ}$ angular and $200 \mu \mathrm{m}$ vertical step sizes. A $473 \mathrm{~nm}$ blue laser was used which is suitable for excitation since its wavelength is close to the peak of the GFP excitation curve which is the fluorescence target in this experiment. The data was collected for all measurement points and stored in the computer. Immediately following the completion of the experiment, the animal was anesthetized with isoflurane, transcardially perfused with $0.2 \mathrm{M}$ PBS followed by $10 \%$ buffered formalin. The brain was removed and post-fixed in $10 \%$ formalin for 24 hours before being transferred to $30 \%$ sucrose/PB. Next, the brain was frozen, sliced into coronal sections of $200 \mu \mathrm{m}$ thickness which were mounted on glass microscope slides, and cover slipped with UltraCruz mounting medium (Santa Cruz, CA). Next, we imaged all slices via confocal fluorescence microscope (Olympus Fluoview FV 1200).

Figure 9 shows the mapped measurements for each depth and for each angle with respect to the position of the fiber's main lobe of radiation. The data is presented after interpolating and processing using gamma correction with a gamma coefficient of 6.0. Starting from a 3D reconstruction of a section of the brain shown in panel (a), we can find the exact location of the optical fiber entry point to the brain. Two cross-sections were of specific interest to validate our results. The first cross-section is a slice imaged by the confocal microscopy shown in panel (b). The path of the optical fiber is marked in the image. Maximum fluorescence appears at approximately $800 \mu \mathrm{m}$ away from the fiber and $6.6 \mathrm{~mm}$ deep inside the tissue, based on confocal microscopy images. The data collected by the optical fiber probe, displayed in panel (d), shows that the maximum intensity is occurring at the depth of $6.3 \mathrm{~mm}$. This difference in measuring the coordination of maximum fluorescence protein expression is the result of the angle of the main lobe of radiation which is not completely orthogonal to the fiber's optical axis. Taking the firing angle of $73.81^{\circ}$ into account, we can use simple geometry to compensate for this discrepancy. The second cross-section of interest is the slice of the brain along the depth of maximum expression which is shown in panel (c). The lobe of radiation is initially at the $0^{\circ}$ and as the fiber rotates clockwise collecting measurements every $20^{\circ}$, it detects the nearest distribution of fluorescence at $135^{\circ}$. This agrees with our collected data which shows maximum expression at exactly $135^{\circ}$. While the distribution expands well into $180^{\circ}$, with respect to the initial position at $0^{\circ}$, the area of expression that is of nearest proximity to the optical fiber has the largest contribution to the measurement.

\section{Conclusions}

It is important to consider the position of an optical fiber as well as the direction of light delivery during optogenetic 


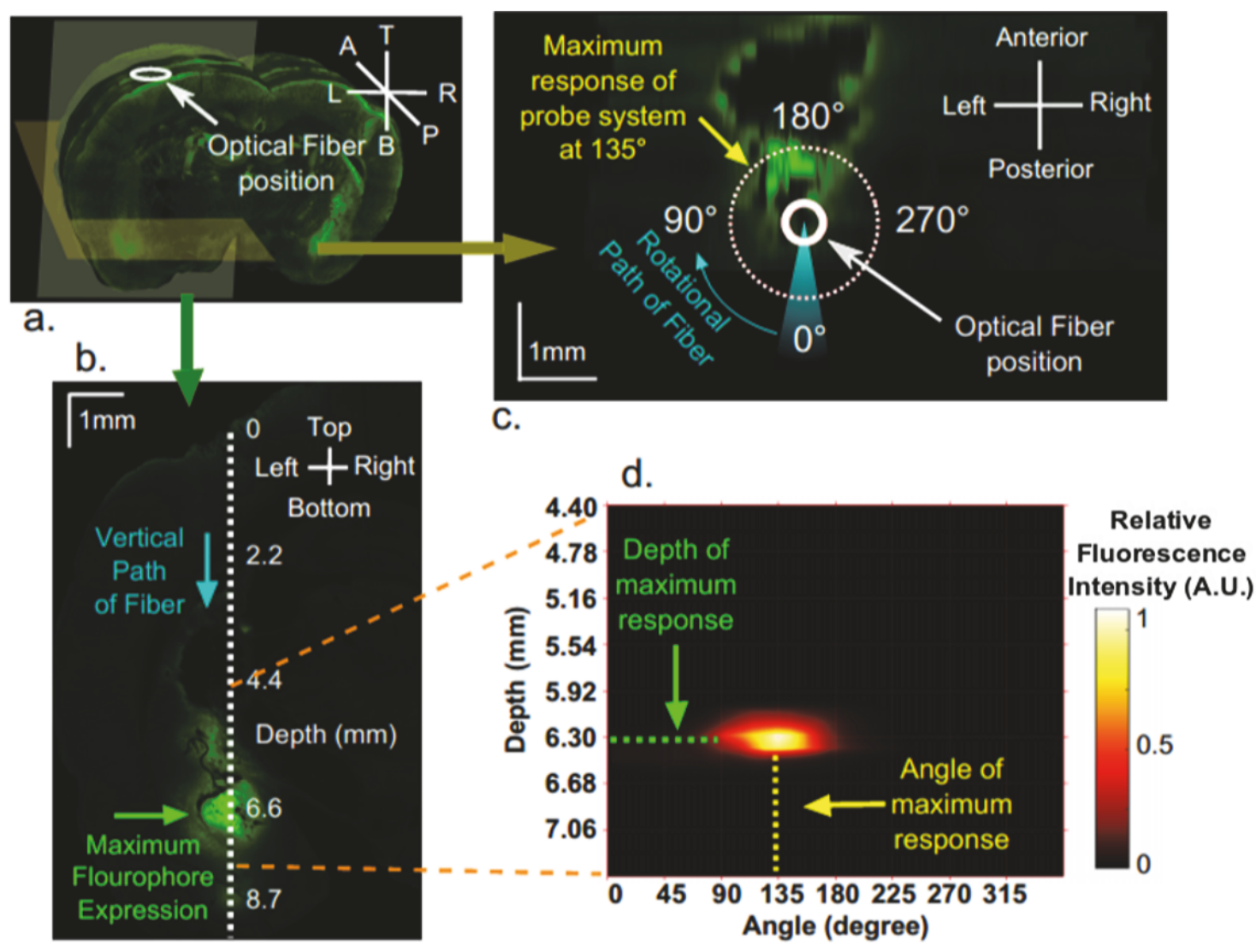

FIGURE 9: (a) In vivo results. 3D image reconstruction of a section of the brain under test. (b) Following the path of the fiber, the maximum expression is found at approximate depth of $6.6 \mathrm{~mm}$ from the surface. (c) Following the rotational path of the fiber, we found the maximum expression closest to the fiber tip at approximately $135^{\circ}$. (d) Data collected with our system and presented using gamma correction factor of 6 .

stimulation $[19,20]$ or other neuroscience experiments. The measured data clearly showed that the expression of fluorescence protein can diffuse in different directions with respect to the fibers main lobe of radiation. With the proposed system, we could accurately detect the depth and the orientation at which the fluorescence protein expression occurs. This information can be used to optimize the light delivery process, for example, in optogenetic experiment to make the optical stimulation more effective or to reduce the amount of optical power necessary to activate targeted neurons and avoid phototoxicity or generating excessive amount of heat in the tissue. While optogenetics is only one application, the proposed system can be used in applications where the distribution of fluorescence needs to be determined in a variety of different soft tissue.

\section{Data Availability}

The data is currently recorded on the lab server. In future, if any potential reader asks for the data, we will be happy to share.

\section{Conflicts of Interest}

The authors declare that there are no conflicts of interest regarding the publication of this paper.

\section{Acknowledgments}

This project was supported by the Army Research Office (ARO) grant\# 68750-LS-II, the National Science Foundation (NSF) Career Award grant\# 1454300, the National Institute of Mental Health (NIMH) grant\# R01MH11241, Brain and Behavior Research Foundation (NARSAD) Young Investigator Award grants\# 20610 and 23620, and the University of Wisconsin research growth initiative (RGI) grants\# 101X213 and 101X254.

\section{References}

[1] T. Vo-Dinh, Biomedical Photonics Handbook, CRC Press, 2003.

[2] D. A. Boas, D. H. Brooks, E. L. Miller et al., "Imaging the body with diffuse optical tomography," IEEE Signal Processing Magazine, vol. 18, no. 6, pp. 57-75, 2001.

[3] E. M. C. Hillman, D. A. Boas, A. M. Dale, and A. K. Dunn, "Laminar optical tomography: Demonstration of millimeterscale depth-resolved imaging in turbid media," Optics Expresss, vol. 29, no. 14, pp. 1650-1652, 2004.

[4] M. Azimipour, M. Sheikhzadeh, R. Baumgartner et al., "Fluorescence laminar optical tomography for brain imaging: System implementation and performance evaluation," Journal of Biomedical Optics, vol. 22, no. 1, 2017. 
[5] R. Pashaie and R. Falk, "Single optical fiber probe for fluorescence detection and optogenetic stimulation," IEEE Transactions on Biomedical Engineering, vol. 60, no. 2, pp. 268-280, 2013.

[6] K. R. Diamond, M. S. Patterson, and T. J. Farrell, "Quantification of fluorophore concentration in tissue-simulating media by fluorescence measurements with a single optical fiber," Applied Optics, vol. 42, no. 13, pp. 2436-2442, 2003.

[7] S. Brooks, C. L. Hoy, A. Amelink, D. J. Robinson, and T. E. Nijsten, "Sources of variability in the quantification of tissue optical properties by multidiameter single-fiber reflectance and fluorescence spectroscopy," Journal of Biomedical Optics, vol. 20, no. 5, Article ID 2290871, 2015.

[8] S. C. Kanick, U. A. Gamm, M. Schouten, H. J. C. M. Sterenborg, D. J. Robinson, and A. Amelink, "Measurement of the reduced scattering coefficient of turbid media using single fiber reflectance spectroscopy: Fiber diameter and phase function dependence," Biomedical Optics Express, vol. 2, no. 6, pp. 1687$1702,2011$.

[9] Q. Liu and N. Ramanujam, "Experimental proof of the feasibility of using an angled fiber-optic probe for depth-sensitive fluorescence spectroscopy of turbid media," Optics Expresss, vol. 29, no. 17, pp. 2034-2036, 2004.

[10] U. A. Gamm, S. C. Kanick, H. J. C. M. Sterenborg, D. J. Robinson, and A. Amelink, "Quantification of the reduced scattering coefficient and phase-function-dependent parameter $\gamma$ of turbid media using multidiameter single fiber reflectance spectroscopy: Experimental validation," Optics Expresss, vol. 37, no. 11, pp. 1838-1840, 2012.

[11] U. A. Gamm, S. C. Kanick, H. J. C. M. Sterenborg, D. J. Robinson, and A. Amelink, "Measurement of tissue scattering properties using multi-diameter single fiber reflectance spectroscopy: In silico sensitivity analysis," Biomedical Optics Express, vol. 2, no. 11, pp. 3150-3166, 2011.

[12] P. R. Bargo, S. A. Prahl, and S. L. Jacques, "Collection efficiency of a single optical fiber in turbid media," Applied Optics, vol. 42, no. 16, pp. 3187-3197, 2003.

[13] T. J. Pfefer, K. T. Schomacker, M. N. Ediger, and N. S. Nishioka, "Multiple-fiber probe design for fluorescence spectroscopy in tissue," Applied Optics, vol. 41, no. 22, pp. 4712-4721, 2002.

[14] I. Diester, M. T. Kaufman, M. Mogri et al., "An optogenetic toolbox designed for primates," Nature Neuroscience, vol. 14, no. 3, pp. 387-397, 2011.

[15] C. Zhu and Q. Liu, "Review of Monte Carlo modeling of light transport in tissues," Journal of Biomedical Optics, vol. 18, no. 5, Article ID 050902, 2013.

[16] L. Wang, S. L. Jacques, and L. Zheng, "MCML-Monte Carlo modeling of light transport in multi-layered tissues," Computer Methods and Programs in Biomedicine, vol. 47, no. 2, pp. 131-146, 1995.

[17] M. Azimipour, R. Baumgartner, Y. Liu, S. L. Jacques, K. Eliceiri, and R. Pashaie, "Extraction of optical properties and prediction of light distribution in rat brain tissue," Journal of Biomedical Optics, vol. 19, no. 7, Article ID 075001, 2014.

[18] Oregon Medical Laser Center, http://omlc.org.

[19] R. Pashaie, P. Anikeeva, J. H. Lee et al., "Optogenetic brain interfaces," IEEE Reviews in Biomedical Engineering, vol. 7, pp. 3-30, 2014.

[20] O. Yizhar, L. E. Fenno, T. J. Davidson, M. Mogri, and K. Deisseroth, "Optogenetics in neural systems," Neuron, vol. 71, no. 1, pp. 9-34, 2011. 

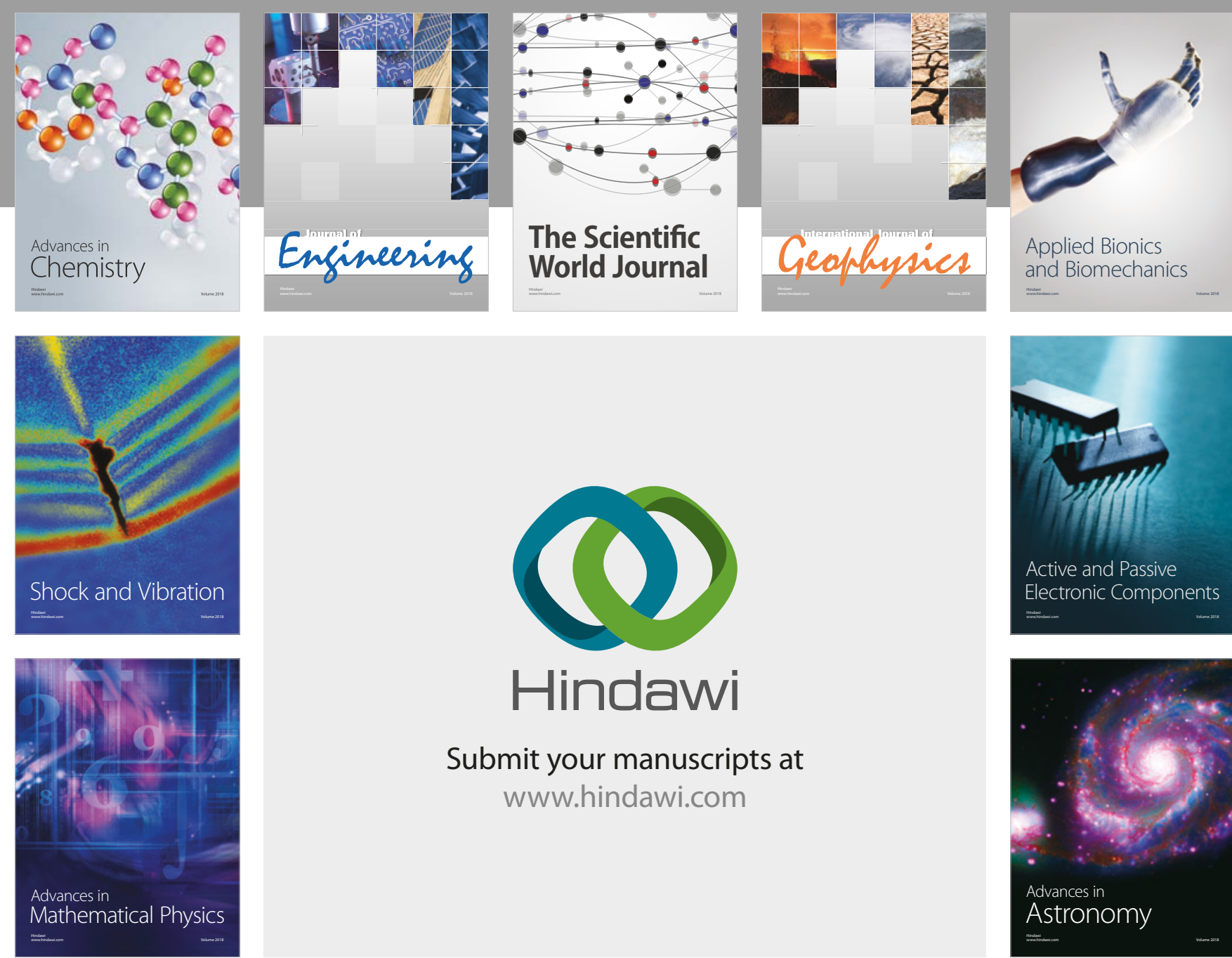

Submit your manuscripts at

www.hindawi.com

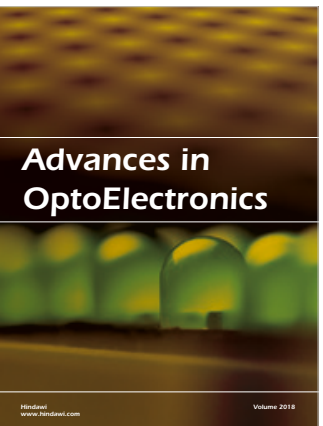

\section{Rotcting Machinery}
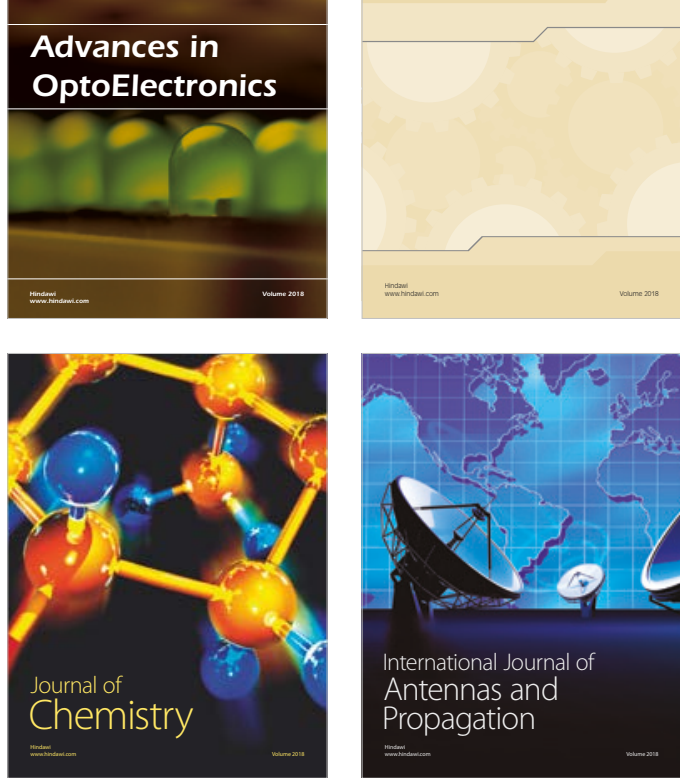

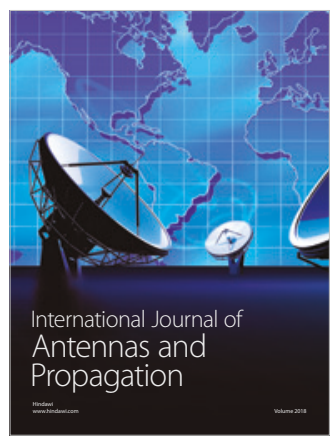

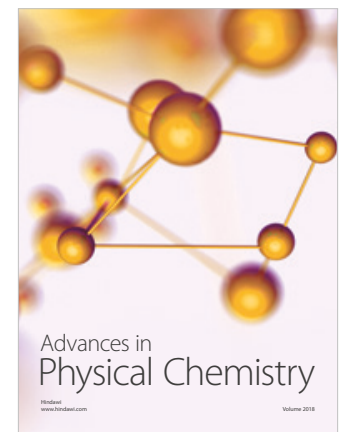

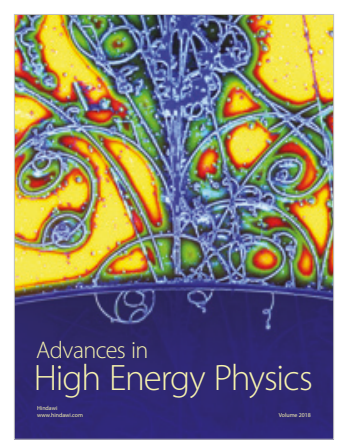

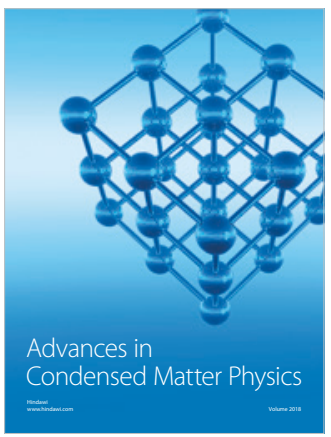

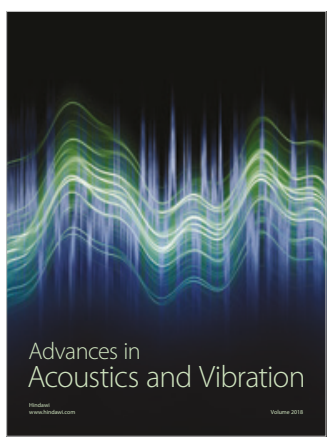

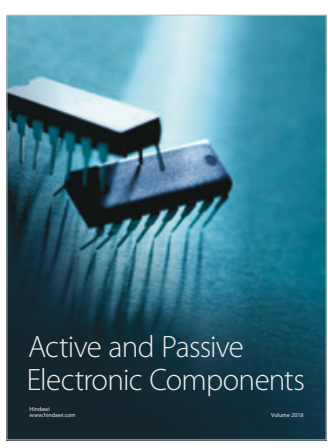
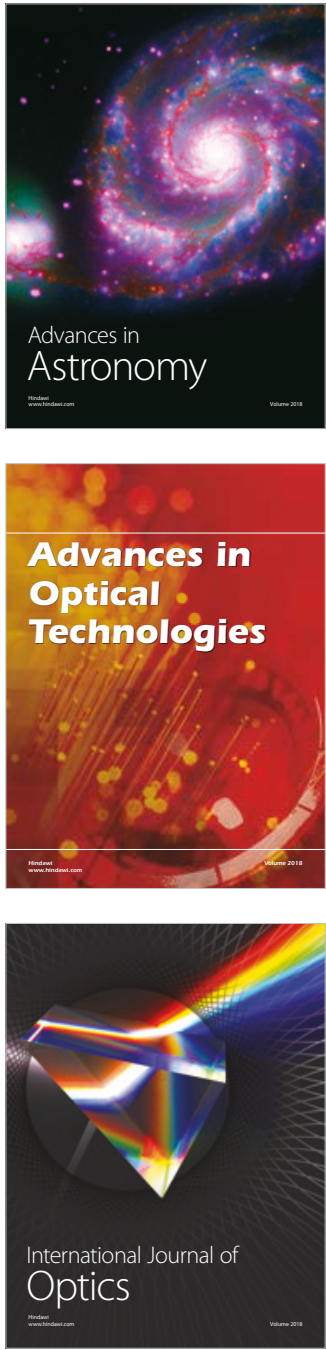